\title{
De 1951 a 1978: un rastreo desde la ruptura con el estructural funcionalismo hasta el materialismo histórico como filosofía de la historia en la vida y obra de Orlando Fals Borda
}

\author{
From 1951 to 1978: a trace from breaking with structural \\ functionalism to historical materialism as a philosophy of history \\ in life and the work of Orlando Fals Borda
}

\section{Resumen}

Daniel Ribero Balaguera*

Recorriendo la obra del maestro como una muestra no sólo de su parábola vital, sino de su evolución paradigmática, el ensayo se sustenta sobre el problema del método científico, como preocupación permanente de Fals. Se indaga sobre la mirada estructural funcionalista del Fals inicial, las limitaciones que encontró en dicho paradigma, los hechos significativos de su vida personal y académica que le plantearon la urgencia de pensar o repensar categorías centrales del análisis sociológico tales como subversión o anti-élites. El ensayo profundiza en la propuesta metodológica y teórica, encaminada a generar una práctica sociológica nueva, propia, que se oponga a la tradicional práctica positivista, dominante en la sociología colombiana y latinoamericana.

Palabras clave: anti-élites, método científico, sociología crítica latinoamericana, sociología positivista, subversión.

\begin{abstract}
Going through the master's work as a sample not only of his vital parable but also of his paradigmatic evolution, this essay bases on the problem of the scientific method, as a permanent concern of Fals. It investigates on the functionalist structural view of the first Fals, the limitations found by him in this paradigm, the significant facts of his personal and academic life that raised the urgency to think or rethink central categories of sociological analysis such as subversion or anti-elites. The essay deepens in the methodological and the theoretical proposal, aimed at generating a new sociological practice, own, that opposes the traditional positivist practice, dominant in Colombian and Latin American sociology.
\end{abstract}

Keywords: panti-elites, latin american critical sociology, positivist sociology, scientific method, subversion.

\section{(i) (1) (2)}

ReCibido: I6 DE JULIO DE 2019 | AprobAdo: I7 DE OCTUBRE DE 2019

\section{Cómo CITAR ESTE ARTICULO}

Ribero Balaguera, D. (2020). De 1951 a 1978: Un rastreo desde la ruptura con el estructural funcionalismo hasta el materialismo histórico como filosofía de la historia en la vida y obra de Orlando Fals Borda. Collectivus, Revista de Ciencias Sociales, 7(1), 51-62. DOI: https://doi.org/10.15648/Collectivus.vol7num1.2020.2531

* Sociólogo, Facultad de Ciencias Humanas de la Universidad Nacional de Colombia, Sede Bogotá. Estudiante de Maestría en Hábitat, Facultad de Artes de la Universidad Nacional de Colombia, sede Bogotá. Correo electrónico: daribero@unal.edu.co 


\section{Introducción}

Cuando presentamos por primera vez este escrito estábamos conmemorando 10 años de la muerte, (el tiempo pasa y ya son dos más) del maestro Orlando Fals Borda. A buena hora y con buen humor muchos lo recuerdan en vida arropados bajo toda una variopinta de sentimientos. Con esto quiero señalar cómo incluso luego de su presunta partida hacia el limbo (¿o quizá se fue hacia el Olimpo?), hoy en día, el maestro tiene la fuerza vital para congregarnos alrededor de su ser-heredado: su legado y la memoria que habita en nosotros.

Si bien es cierto que para los que estamos presentes para este homenaje el maestro Fals es una estrella polar innegable en el trasegar por los caminos de las ciencias sociales, un referente indiscutiblemente nuestro-americano y definitivamente un nombre que se llega a reconocer en las esferas de más altas alcurnias de Colombia, no sobra preguntarnos ¿por qué hemos decidido brindarle un homenaje?

Razones habrá de sobra, junto con sentimientos de zozobra por los tiempos que pasa nuestro país (¡como si eso fuera algo raro!) con los cuales invocamos los indudables motivos para nuestro homenaje. Lo que me ha traído aquí no ha sido él, al contrario, ha sido el fruto de los esfuerzos que se han hecho por omisión, de dejarlo caer en el olvido.

Como todos debemos recordar Orlando Fals Borda y el capellán Camilo Torres fundaron la que en su momento fue la Facultad (hoy reducida a un departamento) de Sociología de la Universidad Nacional de Colombia en 1959. De allí brotaron obras con profundas raíces como La Violencia en Colombia (Fals Borda, 1962) que removieron la tierra sobre los muertos en los que se había asentado el pesado bloque de la clase política sobre la sociedad colombiana.

Es en esta obra, donde el maestro Fals se encuentra no sólo con la cruda realidad de un país convulsionado por la guerra política, también toca un límite del paradigma estructural funcionalista cuando reconoce que dicho marco metodológico e interpretativo utilizado en el estudio de La Violencia (Fals Borda, 1962) no ofrecía suficiencia para "ofrecer explicaciones sociológicas completas del intenso y complicado proceso de la violencia en Colombia" (Fals Borda, 2010, p.431)

Sin embargo, a pesar de este doble descubrimiento científico y social y de los diversísimos aportes que continuó brindando al país y a las gentes explotadas del mundo, ninguno de los padres fundadores del actual departamento de Sociología de la Universidad Nacional tiene una cátedra con nombre propio, tampoco un énfasis metodológico que se encargue de cuidar y desarrollar su legado. En esta situación los hijos del Alma Mater no somos más que un retorcido caso de bastardía, donde no se nos ha permitido reconocer a nuestros padres. Un caso más del abuso al que hemos sido sometidas las nuevas generaciones al obligarnos a vivir en un olvido de nuestra historia.La deuda de la explicación y claridad de los responsables para este olvido forzado, al que nos hemos visto sometidos los estudiantes de sociología de la Nacional, no ha permitido que nuevas semillas crezcan. Hace falta recuperar esas memorias, para sanar las heridas y contribuir a la construcción de nación y democracia en esta linda Colombia.

Abandonando ésta última senda aún perdida para retomar nuestro camino, los siguientes pronunciamientos alrededor del cuestionamiento epistemológico iniciado en 1962 se encuentran en la Subversión en Colombia (Fals Borda, 1967), Por la praxis: cómo conocer la realidad para transformarla (Fals Borda, 1978). Sin embargo, no es sino hasta hacerse obra y síntesis en La Historia doble de la Costa (Fals Borda, 1979)que vemos como Orlando Fals Borda ha decidido adoptar el materialismo histórico como filosofía de la historia (Fals Borda, 1978), como paradigma científico. 
No sobra decir que la ruptura con el estructural funcionalismo en 1962 hasta la adopción del materialismo histórico como filosofía de la historia en 1978-1979 no es un cambio menor. Si bien podemos afirmar que este cambio tiene implicaciones políticas, no podemos afirmar que esas sean sus causas. Es más, si tenemos la osadía de afirmar que la adopción epistemológica de 1978-1979 proviene de una elección política veríamos cómo la falta de prudencia nos lleva a un vacuo empirismo, en donde confundiríamos como elemento esencial lo que en nuestra opinión aparece como verdadero. Así pues, este ensayo es un rastreo de ese camino transitado. Buscamos reconstruir esa trocha abierta, que caminó, sintió y pensó el maestro Fals en vida y de la cual vemos expresión clara en su obra.

\section{El rastreo del problema metodológico en Orlando Fals Borda}

Como debemos saber, más acá que cualquier consideración política, la preocupación del maestro Orlando Fals Borda tuvo como una de sus formas la preocupación sociológica y científica: el problema del método científico.

\subsection{Las primeras obras: el estructural-funcionalismo}

En sus primeras obras sobre el estudio de la realidad colombiana el maestro presta mucha atención a la rigurosidad en el uso del método y las herramientas ya fuera para recopilar la información empírica en el campo, así como para su posterior interpretación. Esta rigurosidad estará presente a lo largo de todas sus obras. Un primer ejemplo de esta rigurosidad que podemos observar aparece en su primera obra "Campesinos de los Andes: estudio sociológico de Saucío" (Fals Borda, 1961). Allí el joven Fals realiza un acercamiento cara a cara con los campesinos saucitas: vive, duerme, come y, lo más importante, habla con ellos como metodología de recolección de información empírica. Luego de recolectada la información es organizada rigurosamente e interpretada siguiendo los marcos de la tendencia que dominaba en la sociología de la época, a saber, el estructural funcionalismo.

Algo parecido sucedió en una de sus obras posteriores. Es en La Violencia en Colombia (Fals Borda, 1962) donde continúa el esfuerzo por el estudio e interpretación de los datos empíricos para dar explicación sociológica de sus observaciones. Esta vez la información guardada por el párroco de El Líbano, Tolima, Germán Guzmán Campos (1912-1988) le fue fundamental para este esfuerzo. El párroco era miembro de la Comisión Gubernamental Investigadora de las Causas de la Violencia, fundada en 1958. En las reflexiones sobre el estudio de todos los datos obtenidos por la Comisión, Fals Borda llega a la conclusión de que el marco metodológico e interpretativo que venía usando - el estructural funcionalismo - no era suficiente para "ofrecer explicaciones sociológicas completas del intenso y complicado proceso de la violencia en Colombia" (Fals Borda, 2010, p.431) Con esto estaba expresando una ruptura explícita con el método usado cuando escribe "sólo se pueden presentar hipótesis y aplicar conceptos en un determinado marco teórico, esperando nuevas técnicas y aportes que permitan establecer cadenas de causalidad y efecto con mayor exactitud" (Ob. Cit.).

La respuesta de censura y crítica sesgada que sufrió la obra en las esferas de poder colombiano tuvo como resultado que Orlando Fals Borda paulatinamente fuera abandonando la relación cercana con el Estado. La confianza en los resultados positivos de las reformas sociales y del alivio que traerían a los problemas rurales y urbanos de Colombia desapareció, al punto de no poder asumir ya la defensa de una perspectiva funcionalista de la sociedad (Moreno, 2017). Con esta obra se cierran los primeros trabajos de Fals Borda, en el cual el paradigma del estructural funcionalismo era la base y el fundamento para su quehacer como sociólogo. 


\subsection{La ida, la vuelta y La Rosca}

Este proceso de búsqueda de alternativas para la sociología en Colombia es una respuesta a lo que se venía gestando en sus obras anteriores. Tal es el caso de la Violencia en Colombia (Fals Borda, 1962), donde se vislumbraban limitaciones sobre el uso del paradigma estructural funcionalista para el análisis del conflicto.

En la búsqueda intelectual que hacía el joven maestro, existe un momento en la vida donde esa radicalidad y preocupación alrededor de la realidad colombiana toman una fuerza que atravesará el resto de su vida y obra. Algunas voces cuentan como la causa de esa fuerza se sostenía en el impacto que tuvo el asesinato de su compañero el Sacerdote Camilo Torres Restrepo en un combate del Ejército Nacional de Colombia

En este trágico momento es cuando sale a la luz la obra la Subversión en Colombia. El cambio social en la historia (Fals Borda, 1967). En ella hay “una compleja elaboración sociológica que introduce el concepto de subversión en la explicación del cambio histórico-social en Colombia desde antes de 1943 hasta 1966. Se podría interpretar que constituye una toma de conciencia intelectual, en el oficio del sociólogo" (Rojas, 2009, p.225). Así (Fals Borda, 1978) define la subversión como "aquella condición o situación que refleja las incongruencias internas de un orden social descubiertas por miembros de éste en un periodo histórico determinado, a la luz de nuevas metas ("utopía") que una sociedad quiere alcanzar" (p.15). Además de introducir el concepto de subversión en la explicación del cambio histórico-social en Colombia" (Rojas, 2009, p.225).

Otro concepto que se construye en la obra de Fals Borda, que será usado en sus escritos, es el de antiélite, que "puede definirse como aquel grupo de personas que ocupando posiciones de alto prestigio se enfrentan a los grupos dominantes para arrebatarle el poder político" (Fals Borda, 1978, p.35). Es papel del científico social entrar a ser parte de esos grupos, ya que la neutralidad valorativa no puede investigar la realidad del cambio sociocultural nuestro-americano, por el contrario "el sociólogo está exigido a comprometerse con la transformación del orden social" (Rojas, 2009, p.226).

La razón primordial de esta posición que adopta un científico, de compromiso con la transformación social y de identificación con los procesos de desarrollo socioeconómico de un país, radica en la constatación de que estos procesos tienen una finalidad o telos, cuya trascendencia y sentido sólo puede aprehenderse mediante la participación activa en ellos. (Fals Borda, 1967, p.275) .

Todo esto llevó a Orlando Fals Borda a desencantarse con la academia al encontrar que los procesos de transformación que él y Camilo Torres veían como necesarios para mejorar las condiciones de vida de los colombianos, no eran posible desde lo que más adelante se convertiría en la Torre de Marfil académica.

Siguiendo a Moreno (2017), luego de este desencanto institucional, Fals Borda decide retirarse del Departamento de Sociología e inicia un viaje a Ginebra en 1968. Allí trabaja con las Naciones Unidas en una investigación global sobre cooperativas en donde trabaja “la hipótesis según la cual los obstáculos al cambio se encontraban fuera de las comunidades, y no dentro de ellas" (p.150), contrario a la hipótesis que manejaba en sus obras previas. Esto lo llevó a considerar más el carácter interno y transformador de los campesinos en sus procesos, junto con las ideas de autonomía de dichos procesos en distintas dimensiones. En su estadía en Ginebra tuvo la oportunidad de conocer las corrientes y redes que desarrollaban críticas al desarrollismo y la sociología que surgía de aquellas críticas. Un viaje a Cuba y a Europa le permiten conocer más cerca los procesos revolucionarios de cambio social (Moreno, 2017) 
Continuando con Moreno (2017) ya hacia el año de 1969 Orlando Fals Borda y su esposa María Cristina Salazar tenían intenciones de regresar a Colombia. Una primera propuesta para realizar a su regreso buscaba rescatar el departamento de Sociología de la Universidad Nacional, sin embargo, luego de consultarlo con sus conocidos en el medio, se dieron cuenta de que por ahí existía mucha resistencia al cambio (jtoda una paradoja!).

Así fue que ambientaron la idea de construir un Centro Colombiano de Estudios Aplicados que buscara servir a la docencia y la investigación multidisciplinar. Frente a esto recibieron dudas y críticas porque lo que pretendían corría muchos riesgos de ser contradictorio con lo que predicarían. Más adelante sus intenciones se materializaron en lo que se llamaría La Rosca de Investigación y Acción Social.

\subsection{Los estatutos de La Rosca de Investigación y Acción social: la centralidad del materialismo histórico}

Tal vez los estatutos de la Fundación La Rosca sean uno de los documentos con mayor riqueza para encontrar las pistas de la adopción del materialismo histórico por parte de Orlando Fals Borda.

El rastreo previo sobre la etapa de la vida de Orlando Fals Borda en la cual adopta explícitamente el materialismo histórico aparece, en un primer momento, en un pie de página de La investigación-acción en la Costa Atlántica: evaluación de La Rosca 1972-1974 (1983), de Ernesto Parra Escobar. En esta evaluación sobre el proceso del trabajo adelantó La Rosca en la Costa Atlántica, se menciona que las razones de la adopción del materialismo histórico se encuentran en las actas de constitución de la Fundación1. Sin embargo, al revisar los documentos preservados en el Archivo Histórico de la Universidad Nacional de Colombia2 se encuentra que la información no está en los estatutos como tal.

El lugar donde aparecen las adopciones teóricas, políticas y metodológicas explícitas alrededor del materialismo histórico aparecen en las "Notas para un programa de la Rosca", documento fechado para octubre de 1971. Allí, en las Notas (Marcuse, 1971) para el programa se hace una alusión directa sobre la decisión de elegir el materialismo histórico en donde se remite a los estatutos de constitución de La Rosca. Los motivos de adopción del materialismo histórico para los miembros de la rosca se expresan de la siguiente manera.

Para los miembros integrantes de La Rosca existen dos procesos "que demandan una respuesta por parte de sectores capacitados para proporcionar una respuesta de alternativas viables, y las demandas de cambio" ya que a pesar de la proliferación de grupos políticos "no es muy clara la gama de alternativas que tales grupos pueden ofrecer a las demandas populares" (Fondo OFB, Caja 51, Folio 72)

El primer proceso es el de la descomposición social, analizado bajo la formación social neo-colonial colombiana y su crecimiento acelerado, con indicadores tan protuberantes como la crisis de cambio internacional, la acelerada descomposición política de la burguesía, el ascenso militar, las alternativas pro-burguesas de Chile, Perú, etc. (Fondo OFB, Caja 51, Folio 72)

El segundo momento es cuando describen como "indudable que las masas colombianas experimentan un creciente ascenso en la vía de adquisición de conciencia política" (Fondo OFB, Caja 51, Folio 72). Es en la adecuación de un método científico para dar respuestas a las demandas de alternativas sociales donde

1 A pesar de que se considera que el material de archivo relacionado con el materialismo histórico se encuentra en Montería junto con los archivos de la Historia Doble de la Costa, el proceso de formación de estas ideas se concentra en la fundación de La Rosca de Investigación y Acción Social.

2 Archivo histórico Universidad Nacional de Colombia. Fondo Orlando Fals Borda. Caja Número 51. 
se encuentran alusiones explícitas a la necesidad de adoptar una actitud mucho más radical3 frente a la realidad colombiana. Tal es el giro hacia una radicalidad epistemológica y el compromiso con la acción que se habla de la necesidad de estudiar la realidad colombiana de manera dialéctica en el estudio de lo abstracto a lo concreto (Fondo OFB, Folio 73), así como del lugar de la Sociología dentro de la nueva adopción epistemológica. Así quedó consignado en el programa de La Rosca:

\begin{abstract}
Tal es el caso de establecer que "sin teoría revolucionaria no hay partido revolucionario, y que sin la preparación adecuada no hay teoría adecuada". Es preciso, por tanto, el análisis científico y riguroso de las condiciones objetivas, sus antecedentes, sus causas y entronque con otros fenómenos a diferentes niveles. Sus relaciones, en fin, a nivel empírico y las posibilidades que esquemas teóricos ofrecen para explicarlas. Por tal razón no basta el simple análisis empírico o positivista (el positivismo ha sido el arma científica más valiosa de la reacción). Es preciso penetrar más allá, en la dialéctica misma de los fenómenos que estudian. En este orden de ideas sería proponer una radación en campos de análisis que viniera de lo abstracto a lo concreto y regresara, no mecánicamente, sino dialécticamente. Si es cierto que sin teoría revolucionaria no hay partido revolucionario, es decir, lucha revolucionaria, el revolucionario no debe temerle al estudio teórico abstracto aparentemente alejado de realidad. Y el estudio empírico de la situación concreta tampoco debe asustarle, ya que es el puente entre la ciencia y la lucha; es la concreción de la lucha revolucionaria. Aceptando estas premisas el Marxismo-Leninismo se nos aparece como la guía teórica más adecuada, la que todo cuadro revolucionario debe conocer. Y la sociología, así mismo, no debe verse más que como un brazo poderoso del materialismo histórico. Esto porque, entre otras cosas, la salida socialista para Colombia es indiscutible y sin marxismo no hay socialismo científico. (Fondo OFB, Caja 51, Folio 73).
\end{abstract}

Así pues, una línea explícita del marxismo-leninismo queda establecida como la línea política y científica que marcará el paso de los siguientes adelantos en la obra de Fals Borda.

\title{
2.4. La maduración del materialismo histórico dentro de la IAP: "Por la Praxis"
}

Luego de todo este intenso proceso social, político y vivencial, Orlando Fals Borda empieza a acercarse a lo que se podría llamar una síntesis conceptual y metodológica que tuvo inicio al terminar de escribir sus reflexiones hacia 1962. Dieciséis años después, en 1978, Fals Borda presenta en el Simposio Mundial de Cartagena sobre Crítica y política en las ciencias sociales, su conferencia “Por la Praxis: El problema de cómo investigar la realidad para transformarla".

El texto de la conferencia de Fals en Cartagena, junto con el de las "Notas para un programa de La Rosca" (Marcuse, 1971; Monreal, Cárdenas y Martínez, 2019) pueden ser los textos que más den cuenta de la importancia del materialismo histórico en la historia de la IAP en Orlando Fals Borda. En las Notas para el programa de 1971 hay una clara intención radical y de carácter más militante alrededor de la teoría y el método del materialismo dialéctico en la investigación sociológica al adoptar el marxismo-leninismo. Frente a las Notas, en "Por la praxis" (Fals Borda, 1978) se presenta la maduración que ha permitido ver los potenciales, los límites alcanzados y las nuevas aperturas y caminos en la investigación sobre la realidad que surgieron durante los dieciséis años mencionados.

\subsection{El problema de cómo investigar la realidad para transformarla (Fals Borda, 1978)}

En esta conferencia Fals Borda delinea, de manera distinta, algunos de los problemas que la sociología positivista, y paradigma en su momento, no podía comprender ni superar para el estudio de los grupos sociales en los cuales había enfocado su investigación de la realidad colombiana. En este apartado sobre la conferencia de 1978 el enfoque será dedicado para explicar, por medio del texto, las razones para la elección

3 Contrario a la experiencia común en la confusión entre radical y extremo como sinónimos, la RAE (Real Academia Española) radical nos muestra que radical tiene una relación con la raíz: 1. adj. Perteneciente o relativo a la raíz. 2. adj. Fundamental o esencial. 3. adj. Total o completo. 
del "materialismo histórico como filosofía de la historia, como punto culminante de unificación" (Fals Borda, 1978, p.12), de las diferentes problemáticas suscitadas por el paradigma estructural-funcionalista.

Como ya habíamos mencionado existen dos dimensiones que empapan toda la obra de Orlando Fals Borda: el estudio de la realidad colombiana y el compromiso del científico social con los grupos explotados y marginados. Las preocupaciones éticas que suscitan ambas dimensiones son fundamentales para comprender el paso definitivo que marca el paso desde el paradigma positivista burgués hace materialismo histórico revolucionario.

Así pues, Fals Borda empieza reconociendo que dentro de los marcos de referencia que se conciben en la ciencia que se elijan deben ser escogido "para nuestros fines, aquello que sea armónico con nuestra visión de responsabilidad social" (Fals Borda, 1978, p.1), puesto que dichos marcos de referencia tienen indudablemente un impacto político4. Por lo tanto, la investigación-acción en Colombia comprende la situación histórico cultural de grupos excluidos, vinculada con la práctica de organizaciones locales y nacionales (Fals Borda, 1978, p.1).

Para resolver inquietudes gnoseológicas alrededor del tema, es decir, los principios fundamentales de la forma en que generamos conocimiento, el marco gnoseológico que expone Fals responde a las necesidades de las dimensiones éticas que componen toda su obra. A saber, una relación entre ser y pensar basada en una conciencia trascendental en donde se genera el conocimiento de una exterioridad con la que se interactúa. Significa partir de que todo el conocimiento, las ideas, pensamientos se dan a partir de un estímulo exterior y la interacción que tienen con las estructuras mentales, sociales, culturales, espirituales e históricas de cada quien. Esta condición, aparentemente nos presenta que sólo podemos conocer nuestros pensamientos generados a partir de esa exterioridad. Sin embargo, esta limitación misma es la posibilidad de que avancemos en lo que producimos como conocimiento científico-social a partir de dichos estímulos externos.

Lo esencial de este conocimiento no es el status o importancia de lo conocido, sino el razonamiento dialéctico que permita llegar a conocer lo desconocido. Esta última dimensión tiene una implicación inseparable para el conocimiento sociológico como lo plantea Fals: la práctica. En todo este proceso de razonamiento y práctica va implicado el proceso de constatación (Fals Borda, 1978). Este proceso de constatación es el ir y volver entre los procesos de análisis y dialéctica trascendental y la puesta a prueba de ellos en la vida misma, en el campo político y de acción social.

Luego de estas aclaraciones preliminares, Fals pasa a esbozar un análisis de las dificultades y límites del paradigma positivista en sus investigaciones realizadas, es decir, la relación entre ciencia y realidad. Arranca con la problemática de uno de los conceptos fundamentales en el pensamiento positivista y las ciencias naturales: la causalidad frente al estudio de los movimientos sociales en Colombia.

A pesar de que en las ciencias sociales se creía "que el mismo concepto de causalidad podría aplicarse en las ciencias naturales como en las sociales" y de que los movimientos sociales según dichos "cánones positivista, pueden ser respuesta a impulsos aplicados en determinados sectores del sistema social; o en efecto de situaciones patológicas" que se pueden modificar (Fals Borda, 1978, p.4), "el estudio más profundo e independiente de los problemas económicos y sociales dejaba traslucir una redes de causas y efectos" que sólo podían ser explicados por modelos que no respondían al paradigma vigente (Fals Borda, 1978, p.5),

4 Esta discusión sobre el papel social y político de los marcos de referencia en las ciencias sociales se puede encontrar a lo largo de su trabajo en obras como "Ciencia y compromiso" (1967), "Pensamiento científico y colonialismo intelectual" (1970) y "El problema de la autonomía científica y cultural en Colombia" (1970). Incluso se puede hablar que con estos trabajos Fals Borda es uno de los pioneros latinoamericanos en abordar las problemáticas sociales de las ciencias humanas derivadas de nuestra condición colonial. 
a saber el estructural funcionalismo. Incluso, ya se podían observar que los procesos correspondían a una "concatenación circular o espiral" con su propio desenvolvimiento y dinámica (Fals Borda, 1978, p.5).

Además de esta limitación del concepto de causalidad heredado de las ciencias naturales, el elemento humano, la aleatoriedad y “determinación múltiple dentro del proceso o marco en el cual adquiere sentido”5 basada en la voluntad, no permitía ubicar la causa de la acción bajo la lectura del positivismo, es decir, la causa basada de la acción en una determinación única. Esta concepción de la acción como múltiples determinaciones que determinan y a su vez son determinadas por una gran cantidad de variables llevan a recuperar que la historia es la escrita por los seres humanos, es decir "la construcción consciente del futuro" (Fals Borda, 1978, p.6).

El siguiente elemento que trata Fals Borda son las diferencias entre la constatación entre las ciencias naturales y las ciencias sociales. Mientras que las ciencias sociales se mantienen en la objetividad y neutralidad de un observador externo, en las ciencias sociales el observador "forma parte del universo a estudiar" (Fals Borda, 1978, p.6), cosa que ya había aprendido Fals Borda en su experiencia en Saucío. Esta razón de ser del observador como miembro y parte del universo tiene que ver con el compromiso de saber que son las masas las protagonistas del proceso. Sin embargo, estos procesos de constatación por medio de la inserción quedan reducidos a la verificación dentro de círculos cerrados de miembros del proceso que comparten relativamente el mismo nivel intelectual (Fals Borda, 1978, p.7). En último término Fals Borda comprende que no son algunos miembros los que validan los resultados, y en este caso el maestro no duda en citar a Mao Tse-Tung y nos presenta la forma de validación: "se determinó la validez del conocimiento por los resultados objetivos de la práctica social y política y no mediante apreciaciones subjetivas" (Mao, 1968a, p.319).

De esta misma manera, el contacto y el diálogo con las personas lo llevó a encontrar que el investigador es al mismo tiempo sujeto (en la práctica) y objeto (en la reflexión) dentro del proceso de investigaciónacción permitiendo una apreciación nueva sobre el papel de las técnicas de recolección empírica sobre el terreno. En el proceso de investigación-acción el maestro Fals encontró que existe una inserción por ritmos, lo que llevaba inevitablemente a que las técnicas quedaran supeditadas a los grupos con los que se actuaba. En esta interacción y en el compartir los propósitos comunes con los grupos en los cuales Fals trabajó, herramientas como la encuesta, el cuestionario o la entrevista recibieron un nuevo sentido, tal fue el caso de las entrevistas en donde un abordaje más participativo permitía romper la dicotomía entre entrevistador y entrevistado (Fals Borda, 1978, p.7). En último término se trata de "ajustar herramientas analíticas a las necesidades reales de las bases y no de los investigadores" (Fals Borda, 1978, p.8).

En todas estas revelaciones de la limitación del paradigma positivista para el estudio de las realidades en el campo de los movimientos sociales con los cuales Fals Borda estaba comprometido, el estudio de la realidad objetiva se enriqueció en su complejidad. Se encontró que los cortes seccionales de los hechos recomendados para el estudio de la realidad desde el paradigma positivista no eran suficiente puesto que pudo observarse que los "hechos quedaban amputados de su dimensión procesual" la cual era parte constitutiva de los hechos mismos (Fals Borda, 1978, p.8). Al interpretarlos de manera procesual llegaban al nivel de entendimiento de los grupos con los que se investigaba al asimilarlos como "cosas para nosotros" (Fals Borda, 1978, p.8). Dos ejemplos muy concretos de este proceso de apropiación del conocimiento generado en la investigación se expresan en lo siguiente en palabras de Fals (1978):

5 Vale la pena anotar algunos comentarios que hace el profesor Jorge Enrique González sobre las implicaciones hermenéuticas implícitas en la IAP de Fals Borda. Un abordaje de algunas de esas relaciones se encuentra en "Fundamentos hermenéuticos de cuatro métodos sociológicos contemporáneos" (2013). 
Uno de los dirigentes campesinos que plasmaron formalmente su ideología, logró explicar en términos de "lucha inconsciente de clase" determinadas pautas tradicionales de la conducta de los terrajeros a cuya base pertenecía. Y el recuerdo de la organización campesina que se había dado en una región hacía caso medio siglo, resurgió como "cosa para nosotros"6, una vez que se tradujo al contexto de las confrontaciones actuales y los viejos luchadores fueron recolocados en el proceso histórico vivo. (p.8)

Así pues, el uso de los conceptos cambia. Ya no se trata de una "teoría fetiche"7 como la nombra Fals Borda, sino al contrario, "no estamos constatando nada nuevo, en efecto, los conceptos, las definiciones y las leyes, aunque necesario para ligar la realidad observada a la articulación intelectual, es decir, para fundamentar las representaciones de la realidad, tienen un valor limitado y circunscrito a contextos determinados para explicar eventos y procesos". Esto llevó a buscar soluciones teóricas para generar conceptos más cercanos a la realidad estudiada en un proceso de ida y venida sobre la teoría a la realidad:

$\leadsto$ Propiciamiento de intercambio entre los conceptos conocidos o pre-conceptos y los hechos o percepciones con observaciones adecuadas del medio social.

$\leadsto$ Siguiendo con la acción a nivel de base para constatar la realidad el medio lo que se quería conceptualizar.

$\gg$ Retornando a reflexionar sobre este conjunto experimental para deducir conceptos más adecuados y obtener mejores luces sobre viejos conceptos o teorías que asi se adaptaron al contexto real.

$\gg$ Volviendo a comenzar el ciclo de investigación para culminarlo en la acción.8

Con estos antecedentes de las limitaciones tanto epistemológicas como políticas del paradigma positivista se justifica el trabajo sobre una ciencia social crítica. La gota que derramó la copa fue el hallazgo en el trabajo de campo de la importancia de la historia para comprender la realidad objetiva9 que mostró que el paradigma positivista no puede contener las complejas realidades de nuestras vivencias. Así pues, fue necesario generar un concepto nuevo de ciencia, que se pasó a llamar la "ciencia popular" en el cual, el materialismo histórico, como filosofía de la historia, brindaba el punto culminante de unificación de todos estos nuevos momentos de investigación que había generado el trabajo con los movimientos sociales (Fals Borda, 1978), además de los instrumentos políticos que brinda esta forma de investigación.

Con lo anterior, podemos atrevernos a decir que Fals ha justificado la necesidad que impone la realidad sobre el paradigma positivista y queda demostrado, por la experiencia en la vida y obra del maestro, la necesidad de un cambio del método para el estudio de la realidad desde los movimientos sociales en Colombia.

\section{Conclusiones}

Este ensayo parte de una pregunta que todo estudiante del Departamento de Sociología de la Universidad Nacional de Colombia en Bogotá se realiza durante la carrera: ¿qué pasa en Colombia? El trasegar por los diferentes semestres y las diferentes asignaturas en el actual pensum del departamento es un camino importante y que sienta bases para ayudar a analizar la realidad. Sin embargo, la ausencia del pensamiento

$6 \quad$ Citando a Lenin, Fals Borda nos recuerda: “Esta transformación de cosas en sí en cosas para nosotros según Lenin, es precisamente el conocimiento" (Lenin, 1974: 110, 111, 179).

7 La tendencia a "absolutizar las leyes y los conceptos y a convertir las definiciones en dogmas, a hacer de la teoría un fetiche, como objeto de culto supersticioso y excesivo" (Fals Borda, 1978, p. 9).

8 Es importante precisar como el modo de investigación que expresa Fals tiene gran armonía con el planteamiento que hace Dussel $(1985 ; 2006)$ sobre el método utilizado por Marx que aparece como marco teórico de este trabajo.

9 "Convicción que, en verdad, venía de mucho antes, desde los primeros estudios de Saucío en 1955 y en Boyacá en 1857 " (Fals Borda, 1978, p.11). 
de Fals Borda no sólo es clara por la inexistencia de una cátedra bajo su nombre, sino porque no existe aquel pensamiento sintetizador y transformador de realidades que el maestro se esforzó por trabajar.

Mucho se escribe y se comenta desde las aulas sobre la realidad del país, pero esto no es suficiente. Prueba de ello es la incapacidad y la falta de acción organizada del movimiento estudiantil que se mueve como sombra de organizaciones y partidos políticos a los cuales están afiliados. Con esto podemos decir que no existe unidad en el movimiento estudiantil, sino fragmentos de sucursales de los partidos o movimientos políticos dentro de las universidades. Esta situación de falta de autonomía del movimiento estudiantil lo ha llevado a ser sacrificado en nombre de intereses particulares.

Mientras tanto, entre los docentes son contados aquellos valientes que se la juegan por el país desde la academia. Sin embargo, hay otros que aún se manejan desde marcos (neo)funcionalistas y muchos los que no se interesan por participar en un horizonte de acción y esperanza.

Es por esto que siempre existirá el imperativo de la recuperación de la memoria de Fals Borda. Pero no sólo desde el recuerdo, sino desde el olvido mismo. Recuperar la memoria de Fals desde el olvido nos permite entrar en la profundidad de su legado, tanto vital como académico. Es lo que nos permite hacer el esfuerzo para sumergirnos en esos subrepticios de la memoria y desentrañar nuestra historia omitida y aún por contar.

Fruto de ese esfuerzo es este ensayo presentado para conmemorar la memoria del maestro, con excusa de la fecha de su partida hacia el limbo.

Así pues encontramos que en un primer momento Fals Borda estaba absolutamente convencido de las bondades y potencialidades del paradigma funcionalista para conocer la realidad social. Sin embargo, al encontrarse con la brutalidad real de una guerra silenciada por las élites políticas se percata de los límites científicos y políticos de dicho paradigma. Esto lo lleva a empezar a replantearse su posición y el paradigma que trabaja.

Siempre que fallece un ser querido cercano quedamos desubicados. Y esto probablemente no suceda solamente por el shock de la desaparición de ese alguien. A medida que pasa el tiempo vamos encontrando cómo la muerte de aquel ser amado fue un redireccionamiento para ubicarnos en nuestro presente. Tal vez la desaparición del padre Camilo Torres Restrepo, junto con todo lo que se dice que le sucedió a Fals Borda durante sus últimos meses en la facultad que fundó, lo llevaron a emprender un viaje a Ginebra donde tuvo la oportunidad de empezar a mirar el problema de las comunidades bajo una nueva óptica. Allí fue donde inició sus trabajos revalorando el papel de las comunidades para enfrentar sus problemas y enfocar las dificultades, ya no en las limitaciones que tendrían dichas comunidades para la adaptación al modelo exterior a ellas, sino por el contrario, enfocarse en cómo el modelo es incapaz de satisfacer las necesidades de dichas comunidades. Haciendo que el modelo es aquello que necesita ser transformado para ser capaz de integrar a las comunidades sin que pierdan los rasgos que las distinguen social e históricamente a sí mismas tanto como para las otras comunidades: un tema de que la autonomía e identidad son sinónimos de dignidad y libertad (García y Rodríguez, 2015).

Todo este viaje que va desde 1951 hasta 1978 en su dimensión personal, vital y académica le permitió a Fals Borda finalmente convenir en la tesis de que el paradigma del materialismo histórico, entendido como filosofía de la historia es el paradigma más adecuado para comprender todos esos conflictos sociales y poder tramitarlos, de tal manera, que lleguen a buen puerto para satisfacer las necesidades de las comunidades, las cuales, también se transforman por sí mismas en sus procesos de lucha y resistencia. 
Quedará mucho por decir, por investigar y por contar. Lo cierto es que la conmemoración del maestro no sólo debe ser sentida en las fechas propuestas para ello. Siempre está la posibilidad de recordarlo y darle homenaje en nuestro proceder ético y comprometido con los explotados del mundo como profesionales, pero más que todo, en el diario vivir. Siempre profundizar en nuestro interior guiados por esa enseñanza que nos legó en esforzarnos por ser seres sentipensantes.

\section{Referencias bibliográficas}

Fals Borda, O. (1961). Campesinos de los Andes: estudio sociológico de Saucío. Bogota: Universidad Nacional.

Fals Borda, O. (1962). La violencia en Colombia. Bogota: Distribuidora y Editora Aguilar, Altea, Taurus, Alfaguara S.A. Colombia.

Fals Borda, O. (1967). Ciencia y Compromiso. ECO Revista de La Cultura de Occidente, 16-2(92), 181-200.

Fals Borda, O. (1978). Cómo investigar la realidad para transformarla. Bogota: FUNDABCO.

Fals Borda, O. (1979). Historia doble de la Costa. Bogota: Universidad Nacional de Colombia.

Fals Borda, O. (2006). Documentos escritos. Caja 51. Carpeta 1.

Fals Borda, O. (2010). Antología. Bogota: Universidad Nacional de Colombia.

García, J., y Rodríguez, P. (2014). La identidad y lo popular: la identidad popular en tiempos de socialismo. Collectivus, Revista de Ciencias Sociales, 2(1): 4-39. DOI: http://dx.doi.org/10.15648/coll.1.2015.2

Marcuse, H. (1971). Razón y revolución. Madrid: Alianza editorial.

Mao Tse-tung. (1968a). Algunas cuestiones sobre los métodos de dirección. Pekín: Ediciones en Lenguas Extranjeras.

Monreal, M., Cárdenas, R., \& Martínez, B. (2019). Estereotipos, roles de género y cadena de cuidados. Transformaciones en el proceso migratorio de las mujeres. Collectivus, Revista de Ciencia Sociales, 6(1), 83-97.

Moreno, M. (2017). Orlando Fals Borda: Ideas, prácticas y redes (1950-1972). Universidad Nacional de Colombia, Bogota.

Rojas, J. . (2009). La construcción del a IAP. Revista Análisis Político, (67), 224-229. 
